Turkish Journal of Mathematics

http://journals.tubitak.gov.tr/math/

Research Article

Turk J Math

(2019) 43: $355-360$

(c) TÜBİTAK

doi:10.3906/mat-1807-200

\title{
A broken cycle theorem for the restrained chromatic function
}

Aysel EREY*(1)

Department of Mathematics, Faculty of Science, Gebze Technical University, Kocaeli, Turkey

$\begin{array}{llll}\text { Received: } 29.07 .2018 & \text { - } & \text { Accepted/Published Online: } 17.12 .2018 \quad \text { Final Version: } 18.01 .2019\end{array}$

\begin{abstract}
A restraint $r$ on a graph $G$ is a function that assigns each vertex of the graph a finite subset of $\mathbb{N}$. For each vertex $v$ of the graph, $r(v)$ is called the set of colors forbidden at $v$. A proper coloring of $G$ is said to be permitted by a given restraint $r$ if each vertex $v$ of the graph receives a color that is not from its set of forbidden colors $r(v)$. The restrained chromatic function, denoted by $\pi_{r}(G, x)$, is a function whose evaluations at integer $x$ values count the number of proper $x$-colorings of the graph $G$ permitted by the restraint $r$ and this function is known to be a polynomial function of $x$ for large enough $x$. The restrained chromatic function $\pi_{r}(G, x)$ is a generalization of the well-known chromatic polynomial $\pi(G, x)$, as $\pi_{r}(G, x)=\pi(G, x)$ if $r(v)=\emptyset$ for each vertex $v$ of the graph. Whitney's celebrated broken cycle theorem gives a combinatorial interpretation of the coefficients of the chromatic polynomial via certain subgraphs (the so-called broken cycles). We provide an extension of this result by finding combinatorial interpretations of the coefficients of the restrained chromatic function.
\end{abstract}

Key words: $x$-Coloring, restraint, chromatic polynomial, restrained chromatic function

\section{Introduction}

In this article all graphs are finite, simple, and undirected. Given a graph $G$, let $V(G)$ be the vertex set of $G$ and $E(G)$ be the edge set of $G$. The order and size of $G$ are $|V(G)|$ and $|E(G)|$, respectively. For a positive integer $x$, an $x$-coloring of $G$ is a function $c: V(G) \rightarrow\{1, \ldots, x\}$ and it is called a proper $x$ - coloring of $G$ if $c(u) \neq c(v)$ for every edge $u v$ in $E(G)$. The well-known chromatic polynomial of $G$, denoted by $\pi(G, x)$, is the polynomial whose evaluations at positive integer $x$-values count the number of proper $x$-colorings of $G$.

In generalized graph coloring problems, each vertex has a list of available or forbidden colors and the goal is to properly color the graph obeying the restrictions. They arise naturally in the regular graph coloring problem because the assignment of some color to each vertex imposes restrictions on the neighbor vertices. Such problems are also useful tools in modeling real-world problems such as scheduling and timetabling [13] and they have been extensively studied in the literature; see, for example, [1, 19]. Here we shall focus on restrained colorings. In a restrained coloring, there is a restraint function $r$ that assigns each vertex of the graph a finite subset of $\mathbb{N}$. For each vertex $v$ of the graph, $r(v)$ is called the set of colors forbidden at $v$. An $x$-coloring $c$ of a graph $G$ is said to be permitted by the restraint $r$ if $c(v) \notin r(v)$ for every vertex $v$ in $V(G)$. The study of restrained colorings proved useful in the construction of critical graphs [17] and recently several extremal problems on this topic were studied in $[4,5,11]$.

*Correspondence: aysel.erey@gtu.edu.tr

2010 AMS Mathematics Subject Classification: 05C15, 05C30, 05C31 
EREY/Turk J Math

Counting the number of generalized colorings has also been studied in a number of settings. Enumeration of list colorings was studied and relations to the chromatic polynomial were investigated in [10, 16, 20]. Recently, Knox and Mohar (arXiv:1708.01781) proved a long-standing conjecture on the chromatic polynomial by studying a list coloring function. Given a positive integer $x$, let $\pi_{r}(G, x)$ be the number of proper $x$-colorings of the graph $G$ permitted by restraint $r$. It is known that $\pi_{r}(G, x)$ is a polynomial function of $x$ when $x$ is large enough, or more precisely, when $x>\max \cup_{v \in V(G)} r(v)$ (see Theorem 4.1.2, p. 102 in [11]). Furthermore, $\pi_{r}(G, x)$ is a monic polynomial of degree $|V(G)|$ with integer coefficients that alternate in sign [11]. We call $\pi_{r}(G, x)$ the restraint chromatic function of $\mathrm{G}$ with respect to the restraint $r$. It is clear that the restraint chromatic function generalizes the chromatic polynomial because $\pi_{r}(G, x)=\pi(G, x)$ if $r(v)=\emptyset$ for every vertex $v$ of $G$.

Since the restrained chromatic function $\pi_{r}(G, x)$ turns out to be a polynomial function of $x$ for large enough $x$, a natural problem is to find a combinatorial interpretation of the coefficients and such interpretation is of crucial importance to study this function. A celebrated result due to Whitney gives a combinatorial interpretation of the coefficients of the chromatic polynomial via the so-called broken cycles (Theorem 2.1). Our main result (Theorem 2.2) generalizes this result to a restrained chromatic function by providing a combinatorial interpretation for its coefficients.

\section{The broken cycle theorem}

A subgraph $H$ of a graph $G$ is called a spanning subgraph of $G$ if $V(H)=V(G)$. A spanning forest of a graph $G$ is a spanning subgraph that is a forest. Given a graph $G$, let $\mathcal{C}(G)$ denote the family of all connected components of $G$. Given a set $S$ and an integer $k$, let $\left(\begin{array}{l}A \\ k\end{array}\right)$ denote the family of all subsets of $A$ with size $k$. Also, let $M_{r}(G)$ be equal to $\max \bigcup_{v \in V(G)} r(v)$ if it is nonempty and 0 otherwise.

Let $f$ be a labeling of the edges of the graph $G$ such that $f: E(G) \rightarrow\{1,2, \ldots,|E(G)|\}$ is a bijection. A path $P$ in the graph $G$ is called a broken cycle with respect to $f$ if $P$ is obtained from a cycle by removing the edge of the maximum label in the cycle. In other words, there is a cycle $C$ and an edge $e \in E(C)$ such that $P=C-e$ and $f(e)>f\left(e^{\prime}\right)$ for every edge $e^{\prime}$ in $E(C) \backslash\{e\}$; see the Figure. The family of subgraphs of a graph that do not contain any broken cycle forms an abstract simplicial complex, known as the broken circuitcomplex. Such a complex has received considerable attention and has been investigated from combinatorial algebraic, topological, and geometric points of view $[2,6,7,14,15]$.

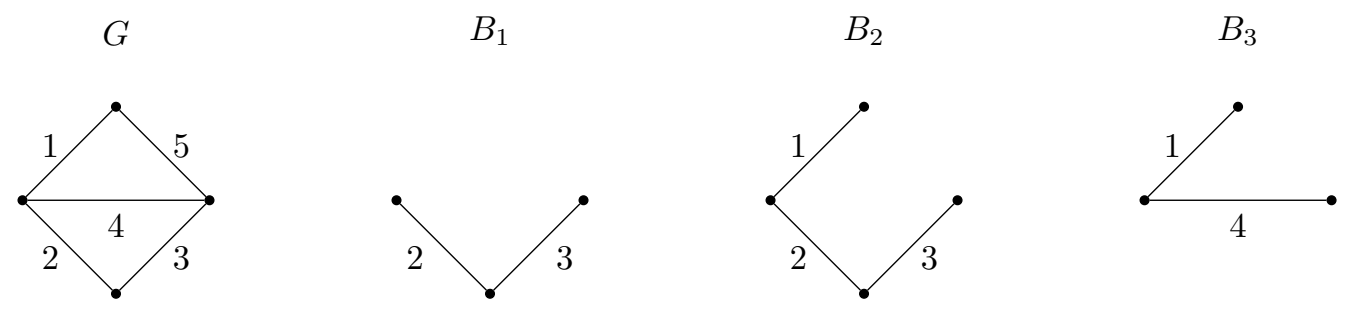

Figure. A graph $G$ with an edge labeling $f$ and its broken cycles $B_{1}, B_{2}$, and $B_{3}$ with respect to $f$.

Theorem 2.1 [21] [Whitney's broken cycle theorem] Let $G$ be a graph on $n$ vertices and $m$ edges, and $f: E(G) \rightarrow\{1,2, \ldots, m\}$ be a bijection. Then 


$$
\pi(G, x)=\sum_{k=1}^{n}(-1)^{k} a_{k}(G) x^{n-k}
$$

where $a_{k}(G)$ is the number of spanning forests of $G$ with size $k$ that do not contain any broken cycle with respect to $f$.

Whitney's broken cycle theorem is a classical result in the theory of chromatic polynomials and several generalizations of this theorem were obtained via various other structures, such as lattices [3], two-variable generalization of the chromatic polynomial [8], matroids (Grinberg, arXiv:1604.03063), and hypergraphs [18]; see [9] for more related work. Our main result is the following, which generalizes Theorem 2.1 via restrained colorings.

Theorem 2.2 Let $G$ be a graph on $n$ vertices, $r$ be a restraint on $G$, and $x>M_{r}(G)$. Then,

$$
\pi_{r}(G, x)=\sum_{k=0}^{n}(-1)^{k} a_{k}(G, r) x^{n-k}
$$

and

$$
a_{k}(G, r)=\sum_{t=0}^{k} \sum_{H \in \mathcal{B} \mathcal{C}^{t}} \sum_{\mathcal{A} \in\left(\begin{array}{c}
\mathcal{C}(H) \\
k-t
\end{array}\right)} \prod_{C \in \mathcal{A}}\left|\bigcup_{v \in V(C)} r(v)\right|
$$

where $\mathcal{B C}^{t}$ is the family of spanning forests of $G$ with size $t$ that do not contain any broken cycle with respect to $f$.

Observe that Theorem 2.2 reduces to Theorem 2.1 when $r(v)=\emptyset$ for every vertex $v$ of $G$. In that case, $\left|\bigcup_{v \in V(C)} r(v)\right|=0$ in the formula for $a_{k}(G, r)$. Note that $\prod_{C \in \mathcal{A}} 0$ is 1 if $\mathcal{A}=\emptyset$ and it is 0 otherwise. Also, $\mathcal{A}=\emptyset$ if and only if $t=k$. Thus, when $r(v)=\emptyset$ for each vertex $v, a_{k}(G, r)=\sum_{H \in \mathcal{B C}^{k}} 1$, which is precisely the number of spanning forests of size $k$ that do not contain any broken cycle.

The broken cycle interpretation of the coefficients has been invaluable in the study of chromatic polynomials. It proved to be a useful tool to understand significant properties of chromatic polynomials. For example, a long-standing log-concavity conjecture was settled by applying algebro-geometric tools to the broken circuit complex [12]. The restrained chromatic function has not been studied as much as the chromatic polynomial and

it has not been well understood yet. There are various aspects of this function to be explored and we believe that Theorem 2.2 may provide insights into a better understanding of the properties of the restrained chromatic function.

\section{Proof of the main result}

Lemma 3.1 Let $G$ be a graph, $r$ be a restraint on $G$, and $x>M_{r}(G)$. Then,

$$
\pi_{r}(G, x)=\sum_{t=0}^{m}(-1)^{t} \sum_{H \in \mathcal{S}(G)^{t}} \prod_{C \in \mathcal{C}(H)}\left(x-\left|\bigcup_{v \in V(C)} r(v)\right|\right)
$$

where $\mathcal{S}(G)^{t}$ is the family of spanning subgraphs of $G$ that have size $t$. 
Proof Let $e_{1}, \ldots, e_{m}$ be the edges of $G$. Also, let $A_{i}$ denote the set of all $x$-colorings of $G$ permitted by $r$ such that the vertices of the edge $e_{i}$ receive the same color. The total number of $x$-colorings of $G$ permitted by $r$ is $\prod_{v \in V(G)}(x-|r(v)|)$. The number of $x$-colorings of $G$ permitted by $r$ that are not proper is equal to $\left|A_{1} \cup \cdots \cup A_{m}\right|$. Therefore, the number of proper $x$-colorings of $G$ permitted by $r$ is

$$
\pi_{r}(G, x)=\prod_{v \in V(G)}(x-|r(v)|)-\left|A_{1} \cup \cdots \cup A_{m}\right| .
$$

By the principle of inclusion-exclusion, we get

$$
\pi_{r}(G, x)=\prod_{v \in V(G)}(x-|r(v)|)+\sum_{t=1}^{m}(-1)^{t} \sum_{1 \leq i_{1}<\cdots<i_{t} \leq m}\left|A_{i_{1}} \cap \cdots \cap A_{i_{t}}\right| .
$$

Consider $t$ edges of $G$, without loss, say $e_{1}, \ldots, e_{t}$. Let $H$ be the spanning subgraph of $G$ whose edge set is $\left\{e_{1}, \ldots, e_{t}\right\}$. Now $\left|A_{1} \cap \cdots \cap A_{t}\right|$ is precisely the number of $x$-colorings of $G$ permitted by $r$ such that each connected component of $H$ is monochromatic, i.e. the vertices in the same connected component of $H$ receive the same color. Thus,

$$
\left|A_{1} \cap \cdots \cap A_{t}\right|=\prod_{C \in \mathcal{C}(H)}\left(x-\left|\bigcup_{v \in V(C)} r(v)\right|\right)
$$

and

$$
\sum_{1 \leq i_{1}<\cdots<i_{t} \leq m}\left|A_{i_{1}} \cap \cdots \cap A_{i_{t}}\right|=\sum_{H \in \mathcal{S}(G)^{t}} \prod_{C \in \mathcal{C}(H)}\left(x-\left|\bigcup_{v \in V(C)} r(v)\right|\right) .
$$

Hence, the result follows.

Now using Lemma 3.1 we shall derive the next result.

Lemma 3.2 Let $G$ be a graph of order $n$ and size $m, r$ be a restraint on $G$, and $x>M_{r}(G)$. Also, let $f: E(G) \rightarrow\{1, \ldots, m\}$ be a bijection. Then,

$$
\pi_{r}(G, x)=\sum_{t=0}^{n-1}(-1)^{t} \sum_{H \in \mathcal{B C}^{t}} \prod_{C \in \mathcal{C}(H)}\left(x-\left|\bigcup_{v \in V(C)} r(v)\right|\right),
$$

where $\mathcal{B C}^{t}$ is the family of spanning forests of $G$ with size $t$ that do not contain any broken cycle with respect to $f$.

Proof Let $B_{1}, \ldots, B_{k}$ be broken cycles in $G$ for some $k \geq 0$. Also let $e_{1}, \ldots, e_{k}$ be the edges of $G$ such that $e_{i} \notin E\left(B_{i}\right)$ and $B_{i}+e_{i}$ is a cycle in $G$ for each $i=1, \ldots, k$. Given a broken cycle $B$, we define $\mu(B)=\max \{f(e): e \in E(B)\}$. We may assume that $\mu\left(B_{1}\right) \leq \cdots \leq \mu\left(B_{k}\right)$. Now we partition the family of spanning subgraphs of $G$ into $k+1$ subfamilies as follows. For $1 \leq i \leq k$, let $\mathcal{F}_{i}$ be the family of spanning subgraphs of $G$ that contain $B_{i}$ but do not contain any of $B_{1}, \ldots, B_{i-1}$ (if $i \geq 2$ ). Also, let $\mathcal{F}_{k+1}$ be the 
family of spanning subgraphs of $G$ that contains none of $B_{1}, \ldots, B_{k}$ as a subgraph. It is clear that $\mathcal{F}_{1}, \ldots, \mathcal{F}_{k+1}$ are mutually disjoint and every spanning subgraph of $G$ belongs to some subfamily $\mathcal{F}_{i}$. Let $1 \leq i \leq k$ be fixed. For every subgraph $H$ in $\mathcal{F}_{i}$ that does not contain the edge $e_{i}$, there exists a unique subgraph $H+e_{i}$. It is clear that $H+e_{i}$ also contains $B_{i}$. Moreover, for $2 \leq i \leq k$, the subgraph $H+e_{i}$ does not contain any of $B_{1}, \ldots, B_{i-1}$ because $f\left(e_{i}\right)>\mu\left(B_{i}\right)$ by the definition of broken cycles, and $\mu\left(B_{i}\right) \geq \mu\left(B_{j}\right)$ for each $j=1, \ldots i-1$ by the assumption. Note that $H$ and $H+e_{i}$ have exactly the same connected components and their sizes differ by one. Hence, in the formula for $\pi_{r}(G, x)$ given in Lemma 3.1, the contribution of $H$ and $H+e_{i}$ cancel each other. Thus, the total contribution of each subfamily $\mathcal{F}_{i}$ is zero for $1 \leq i \leq k$. Thus, it suffices to consider the contributions of the subgraphs in $\mathcal{F}_{k+1}$. Since the subgraphs in $\mathcal{F}_{k+1}$ do not contain any broken cycle, they cannot contain any cycle at all. Therefore, $\mathcal{F}_{k+1}$ consists of spanning forests of $G$ that do not contain any broken cycle with respect to $f$ and we obtain the result by Lemma 3.1.

We are now ready to prove our main result.

Proof [Proof of Theorem 2.2] Let $H \in \mathcal{B C}^{t}$ for some $t$. Then $|\mathcal{C}(H)|=n-t$ as $H$ is a spanning forest. Observe that

$$
\prod_{C \in \mathcal{C}(H)}\left(x-\left|\bigcup_{v \in V(C)} r(v)\right|\right)=\sum_{j=0}^{n-t}(-1)^{j} \sum_{\mathcal{A} \in\left(\begin{array}{c}
\mathcal{C}(H) \\
j
\end{array}\right)} \prod_{C \in \mathcal{A}}\left|\bigcup_{v \in V(C)} r(v)\right| x^{n-t-j} .
$$

By Lemma 3.2, $\pi_{r}(G, x)$ is equal to

$$
\sum_{t=0}^{n-1}(-1)^{t} \sum_{H \in \mathcal{B} \mathcal{C}^{t}} \sum_{j=0}^{n-t}(-1)^{j} \sum_{\mathcal{A} \in\left(\begin{array}{c}
(\mathcal{C}(H) \\
j
\end{array}\right)} \prod_{C \in \mathcal{A}}\left|\bigcup_{v \in V(C)} r(v)\right| x^{n-t-j},
$$

which equals

$$
\sum_{t=0}^{n-1} \sum_{j=0}^{n-t}(-1)^{t+j} \sum_{H \in \mathcal{B} \mathcal{C}^{t}} \sum_{\mathcal{A} \in\left(\begin{array}{c}
\mathcal{C}(H) \\
j
\end{array}\right)} \prod_{C \in \mathcal{A}}\left|\bigcup_{v \in V(C)} r(v)\right| x^{n-t-j} .
$$

Now, letting $k=t+j$, we see that the latter is equal to

$$
\sum_{k=0}^{n}(-1)^{k} \sum_{t=0}^{k} \sum_{H \in \mathcal{B C}^{t}} \sum_{\mathcal{A} \in\left(\begin{array}{c}
\mathcal{C}(H) \\
k-t
\end{array}\right)} \prod_{C \in \mathcal{A}}\left|\bigcup_{v \in V(C)} r(v)\right| x^{n-k}
$$

and the result follows.

\section{Acknowledgment}

This work was supported by the Scientific and Technological Research Council of Turkey (TÜBİTAK), research grant $118 \mathrm{C} 009$.

\section{References}

[1] Alon N. Restricted colorings of graphs. In: Proceedings of the 14th British Combinatorial Conference; Cambridge; 1993. pp. 1-33.

[2] Björner A, Ziegler G. Broken circuit complexes: factorizations and generalizations. J Combin Theory Ser B 1991; 51: 96-126. 
EREY/Turk J Math

[3] Blass A, Sagan BE. Möbius functions of lattices. Adv Math 1997; 127: 94-123.

[4] Brown JI, Erey A. Restraints permitting the largest number of colourings. Discrete Appl Math 2017; 222: 76-88.

[5] Brown JI, Erey A, Li J. Extremal restraints for graph colourings. J Comb Math Comb Comput 2015; 93: $297-304$.

[6] Brylawski T. The broken-circuit complex. T Am Math Soc 1977; 234: 417-433.

[7] Brylawski T, Oxley J. Several identities for the characteristic polynomial of a combinatorial geometry. Discrete Math 1980; 31: 161-170.

[8] Dohmen K, Pönitz A, Tittmann P. A new two-variable generalization of the chromatic polynomial. Discrete Math Theor Comput Sci 2003; 6: 69-89.

[9] Dohmen K, Trinks M. An abstraction of Whitney's broken circuit theorem. Electron J Combin 2014; $21: 4.32$.

[10] Donner Q. On the number of list-colorings. J Graph Theory 1992; 16: 239-245.

[11] Erey A. An investigation on graph polynomials. PhD, Dalhousie University, Halifax, Canada, 2015.

[12] Huh J. h-Vectors of matroids and logarithmic concavity. Adv Math 2015; 270: 49-59.

[13] Kubale M. Interval vertex-colouring of a graph with forbidden colours. Discrete Math 1989; 74: $125-136$.

[14] Llamas A, Martínez-Bernal J, Merino C. On the broken-circuit complex of graphs. Comm Algebra 2010; 38: 18471854.

[15] Nakamura M. On the NBC-complexes and $\beta$-invariants of abstract convex geometries. Discrete Appl Math 2009; 157: $1799-1805$.

[16] Thomassen C. The chromatic polynomial and list colorings. J Combin Theory Ser B 2009; 99: $474-479$.

[17] Toft B. Color-critical graphs and hypergraphs. J Combin Theory Ser B 1974; 16: 145-161.

[18] Trinks M. A note on a broken-cycle theorem for hypergraphs. Discuss Math Graph Theory 2014; 34: 641-646.

[19] Tuza Z. Graph colorings with local restrictions - A survey. Discuss Math Graph Theory 1997; 17: 161-228.

[20] Wang W, Qian J, Yan Z. When does the list-coloring function of a graph equal its chromatic polynomial. J Combin Theory Ser B 2017, 122: 543-549.

[21] Whitney H. A logical expansion in mathematics. B Am Math Soc 1932; 38: 572-579. 